\title{
Optic variables used to judge future ball arrival position in expert and novice soccer players
}

\author{
Cathy M. Craig \\ Queen's University Belfast, Belfast, Northern Ireland \\ AND \\ Cédric Goulon, Eric Berton, Guillaume Rao, \\ LAURE Fernandez, AND REINOUd J. BoOtSMa \\ University of the Mediterranean, Marseille, France
}

\begin{abstract}
Although many studies have looked at the perceptual-cognitive strategies used to make anticipatory judgments in sport, few have examined the informational invariants that our visual system may be attuned to. Using immersive interactive virtual reality to simulate the aerodynamics of the trajectory of a ball with and without sidespin, the present study examined the ability of expert and novice soccer players to make judgments about the ball's future arrival position. An analysis of their judgment responses showed how participants were strongly influenced by the ball's trajectory. The changes in trajectory caused by sidespin led to erroneous predictions about the ball's future arrival position. An analysis of potential informational variables that could explain these results points to the use of a first-order compound variable combining optical expansion and optical displacement.
\end{abstract}

Trying to define expertise is a very challenging exercise in sports science. Although most of the literature points to superior perceptual-cognitive skills, such as pattern recognition (Williams \& Ericsson, 2005) and anticipation (Goulet, Bard, \& Fleury, 1989), few studies have described the informational sources used by experts to "read" the game. In fact, most of the research in ball sports has dealt with information pickup in action preparation before ball contact (Abernethy, Gill, Parks, \& Packer, 2001). What happens after ball contact? Are experts better at reading the trajectory of the ball, or does expertise lie in pre-ball-contact perceptual-cognitive processing?

The aim of the present study was not to demarcate the boundaries of expertise in soccer, but rather to identify the informational variables used by experts and nonexperts to predict, from the unfolding trajectory, where the ball is going. More specifically, in the present study we were interested in the phenomenon of spin and its effect on the perception of the ball's future trajectory. Although the effect of spin on ball trajectories has been extensively modeled by physicists (Bray \& Kerwin, 2003; Carré, Asai, Akatsuka, \& Haake, 2002), very little is known about what the person on the receiving end makes of such situations. Although a recent study by Craig, Berton, Rao, Fernandez, and Bootsma (2006) demonstrated that top-class soccer players confronted with free-kicks subjected to spin make systematic judgment errors about where the ball is going, it did not identify the optical variables that underlie those judgments. The aim of the present study was to see whether the judgment errors of experts can be traced to the use of a particular informational variable and whether this informational variable is also used by novices to judge the future arrival position of (spinning and nonspinning) balls.

It is generally assumed that catching or blocking a ball-one that is often traveling at over $70 \mathrm{~km} / \mathrm{h}$ (Morya, Ranvaud, \& Pinheiro, 2003) - in the goalmouth in soccer requires anticipation of the future flight path of the ball. This type of skill ultimately depends on the goalkeeper's detecting the relevant spatial-temporal parameters from a ball's trajectory that specify where it is going (Bootsma, 1991; Regan \& Kaushal, 1994) and when it is going to get there (Lee \& Young, 1985; Peper, Bootsma, Mestre, \& Bakker, 1994). These so-called informational variables have to be prospective in nature, continually informing the actor about the future direction of a moving object and the time remaining until it gets to an interception point. As with any interception task, coordinated action has to be initiated ahead of time. Many studies have thus focused on the temporal aspects of interception - namely, the detection and use of time-to-contact information (Bootsma, Fayt, Zaal, \& Laurent, 1997; Lee, 1998). Fewer studies have focused on the identification of the information, which specifies "where" the object is going to arrive (but see Gray \& Regan, 2006; Harris \& Drga, 2005; Peper et al., 1994).

By manipulating the spin rate, one can effectively alter a projectile's flight path (de Mestre, 1990; Mehta, 1985). Although many other factors, such as the type of surface

C. M. Craig, cathy.craig@qub.ac.uk 
of the sphere or air density, have an effect on how a sphere flies through the air, this study focused on the effects of sidespin that provoked a lateral deviation in a soccer ball's flight path. Craig et al. (2006) reported that such an aerodynamic modulation of the flight trajectory influenced the ability of professional soccer players (both goalkeepers and field players) to anticipate where a ball was going. Experiment 1 extended this study to include a group of novices. Determining whether novices make similar errors in judgment will help us to conclude that we are all sensitive to the same visual information and that expertise may be demarcated more at the level of perception-action coupling than at the level of anticipation.

\section{METHOD}

\section{Trajectories}

A validated aerodynamics model including gravity, drag, and Magnus-Robins lift forces was used to derive ball trajectories (for mathematical details, see supplementary information in Craig et al., 2006). Maintaining magnitude at a constant $36 \mathrm{~m} / \mathrm{sec}$, different trajectories were obtained by varying the direction of the initial velocity vector. Trajectories could arrive (at a height between 1.1 and $1.7 \mathrm{~m}$ ) at eight different lateral positions along the goal line: 0.66, 2.66, 4.66 , and $6.66 \mathrm{~m}$ to the left and to the right of the center of the goal. Given that the goal was $7.32 \mathrm{~m}$ wide, half of the trajectories would enter the goalmouth, whereas the other half would end up going wide. By varying the sidespin (+600 rpm, clockwise spin [CS]; $0 \mathrm{rpm}$, no spin [NS]; and $-600 \mathrm{rpm}$, counterclockwise spin [CCS]), balls could travel from their initial positions to the same given arrival positions following a different trajectory. In Experiment 1, all trajectories started from a single departure point, located $30 \mathrm{~m}$ straight ahead from the goal center (see Figure 1), which - along with the eight different arrival positions and three spin conditions - gave rise to a total of 24 different trajectories. Trajectories disappeared at either 12.5 or $10.0 \mathrm{~m}$ from the goal line, giving rise to 48 different conditions. Ball flight times varied between 0.84 and $0.89 \mathrm{sec}$ in the $10.0-\mathrm{m}$ cutoff conditions, and between 0.73 and $0.77 \mathrm{sec}$ in the $12.5-\mathrm{m}$ cutoff conditions.

\section{Apparatus and Procedure}

For each trajectory, the $x-, y$-, and $z$-coordinates of the ball during the free kick were calculated at 100 times/sec by the model and were saved as an ASCII file. These data were then used to animate, in real time, a black-and-white textured sphere that resembled the color and size of a real soccer ball. The virtual reality animation program, running on a Dell PC equipped with a GForce4 graphics card, placed the sphere $30 \mathrm{~m}$ from the center of the goalmouth in a virtual soccer stadium, whose playing field, goalposts, line markings, and so on conformed to FIFA regulations (see Figure 1). At the beginning of each trial, the ball remained stationary for $0.5 \mathrm{sec}$ to allow the participant to focus on the ball's position. As if struck by an invisible player, the ball then moved according to the spatiotemporal characteristics of the calculated trajectory and disappeared from the display at either 10.0 or $12.5 \mathrm{~m}$ from the goal line.

Participants viewed the virtual soccer stadium through two small screens inside a three-dimensional head-mounted display (HMD) unit (Cybermind Hi-Res900; the field of view was $31.5^{\circ}$ for each eye, and the resolution was $800 \times 600$ pixels). This HMD was equipped with an integrated head-tracking system (Flock of Birds electromagnetic sensor, Ascension Technologies) linking the displayed viewpoint of the virtual world to head orientation (delay $<30 \mathrm{msec}$ ). Participants viewed a full-sized virtual soccer field as if they were positioned in the middle of the goalposts facing the center of the field. During a preliminary exploratory phase, participants were encouraged to turn their heads right, left, up, and down, so as to get their bearings with respect to the goalposts. A training period followed in which participants were made familiar with the task - namely, to judge whether the ball would have entered the goalmouth or not. In order to avoid any learning effects that might arise from seeing the free kicks with spin, participants were presented with only 32 (two cutoff distances, eight arrival positions, presented twice) free kicks without spin. If participants thought that the ball would have gone into the goal, they pressed the left mouse button, and if they thought the ball would have gone wide, they pressed the right mouse button. Performance feedback was given after each response. This session served to familiarize the participants with the buttonpressing procedure and to reinforce their felt position with respect to the goalposts.

During the experimental session, performance feedback was no longer provided. The order of presentation was randomized, and each trajectory was judged 10 times.

\section{Experiment 1}

Participants. A group of 13 non-soccer-players (men between 22 and 31 years of age [NOV-1 group]) was compared with Craig et al.'s (2006) group of 20 players (11 field players [FP-1 group] and 9 goalkeepers [GK-1 group]), drawn from the professional squads of Olympique de Marseille (from France), AC Milan (from Italy), Bayern Munich, and Schalke 04 (both from Germany).

Experimental design. With all balls starting from a single departure point, the combination of eight arrival positions $(0.66,2.66$, 4.66 , and $6.66 \mathrm{~m}$ to the left and to the right of the center of the goal), three spin conditions (CS, NS, and CCS), and two cutoff distances ( 10.0 and $12.5 \mathrm{~m}$ ) gave rise to 48 different conditions that were randomly presented 10 times (for a total of 480 trials/participant).

\section{Experiment 2}

Rationale. Under the conditions of Experiment 1, the ball always departed from the same position, located straight ahead of the center of the goal at which the participants were situated. In order to explore a larger range of conditions, the location of the departure point was varied in Experiment 2.

Participants. Eight non-soccer-players (NOV-2 group), between 24 and 39 years of age, none of whom had participated in the first experiment were tested using exactly the same protocol as in Experiment 1

Experimental design. The ball had two possible departure positions located $4 \mathrm{~m}$ to the left or to the right of the center (at a constant distance of $30 \mathrm{~m}$ from the goal line). The combination of two departure points $(-4$ and $+4 \mathrm{~m}$ ), eight arrival positions (the same as in Experiment 1), three spin conditions (CS, NS, and CCS, as in Experiment 1), and two cutoff distances (10.0 and $12.5 \mathrm{~m}$, as in Experiment 1) gave rise to 96 different conditions. Each participant was confronted 10 times with the same condition, and a total of 960 free kicks were judged.

\section{RESULTS}

\section{Effects of Experience}

Notwithstanding their depth of experience, a strong and systematic influence of the amount and direction of spin was found for the professional soccer players' judgments of whether the ball would end up in the goalmouth or not (Craig et al., 2006). The group of non-soccer-players participating in Experiment 1 revealed very much the same pattern of results (see Figure 1). Indeed, differences between the group of professional players and the group of non-soccer-players remained far from being statistically significant (all $p \mathrm{~s}>.1$ ). The only significant effect involving the factor of group was found for the arrival position $X$ spin condition $\times$ group interaction $[F(28,420)=3.5, p<$ $.001]$. This interaction effect was due to a difference ob- 
A

NS

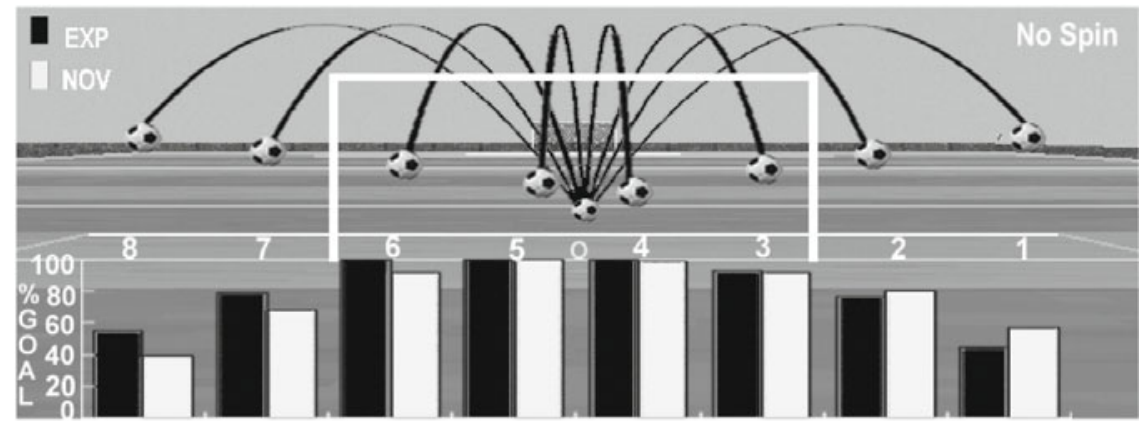

B

\section{CCS}

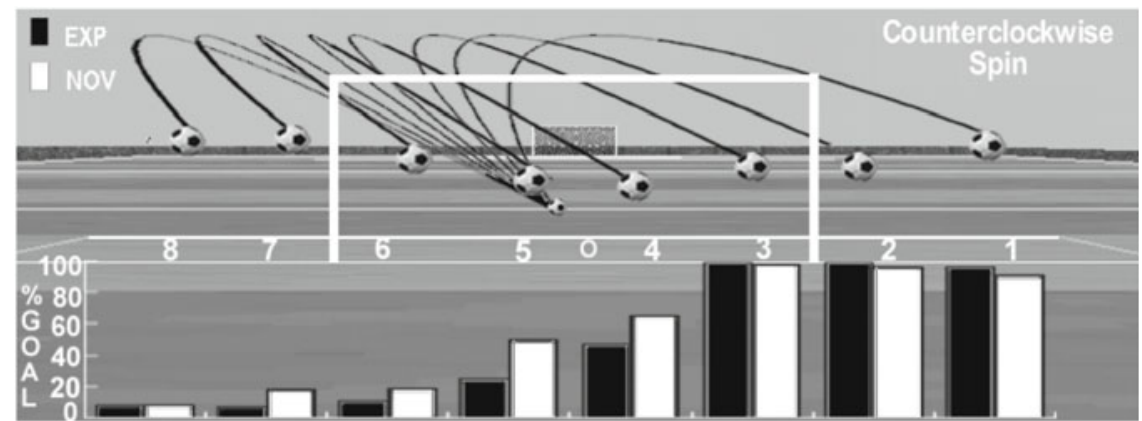

C

CS

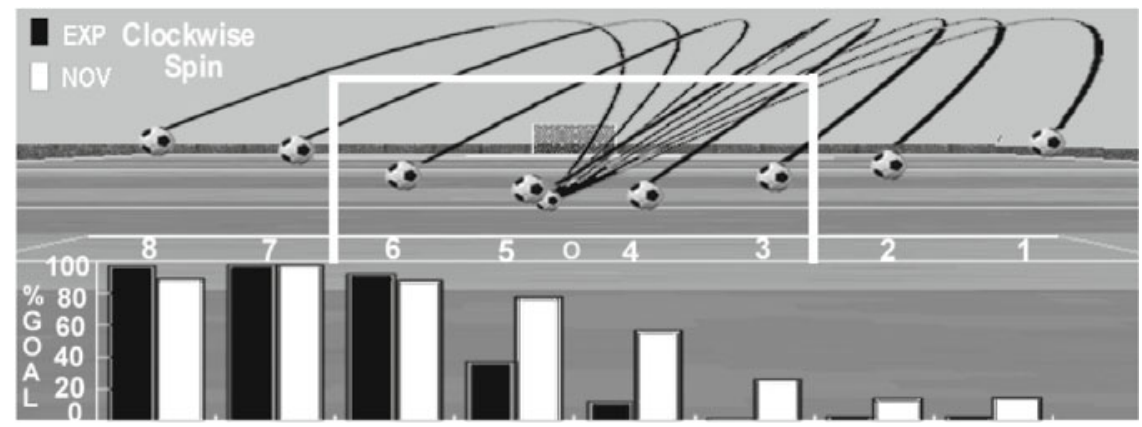

Figure 1. Percentages of goal responses obtained under different conditions of spin for the group of experts (goalkeepers and field players: EXP) and the group of non-soccer-players (NOV) for the eight arrival points (1-8 on the $x$-axis). "O" corresponds to the point of observation. These mean results are for the 10.0-m cutoff condition only (on average, $1.5 \%$ better than the 12.5-m condition), with the thick black lines at the end of the trajectory representing the section that participants did not see. NS, no-spin condition; CCS, counterclockwise-spin condition; CS, clockwise-spin condition.

served in the responses for Positions 4 and 5 in the CS and CCS trajectory conditions, in which non-soccer-players were scoring at chance level ( $42 \%)$, whereas experts were systematically judging the balls to be going wide.

\section{Effects of Trajectories With Sidespin}

When the trajectory of the ball was not subjected to any additional sidespin (NS condition), the percentage of trials that were judged "goal" was symmetrically distributed around the center of the goal and resembled an inverted $\mathrm{U}$
(Figure 1A). The percentage judged "goal" fell from close to $100 \%$ for the central arrival Positions 4 and $5(0.66 \mathrm{~m}$ to the left and to the right of the participant) to around $40 \%$ for the most peripheral positions $[F(7,224)=151.7, p<$ $.001]$. This pattern of results was profoundly influenced by the presence of spin on the ball $[F(2,60)=65.05$, $p<.001$ ], as is highlighted in Figure 1. CCS (Figure 1B) shifted the pattern of results toward the right, with balls arriving at Positions 1, 2, and 3 being judged as goals, whereas balls arriving at Positions 6, 7, and 8 were judged 
as not being goals. The opposite pattern was observed for the CS condition (Figure 1C), with the pattern of results shifting to the left: Balls arriving at Positions 1,2, and 3 were now judged as going wide, whereas balls arriving at Positions 6, 7, and 8 were judged as being goals.

The results from a different group of non-soccerplayers in Experiment 2 (NOV-2) revealed the same pattern of results [significant main effect for spin condition, $F(2,14)=44.19, p<.001]$. A graphic representation of the results for this second experiment can be found in Figure 3 (Columns 1 and 3).

\section{Informational Variables}

In the present series of experiments, we focused on the effects that a ball trajectory subjected to sidespin had on anticipatory judgments. The analysis of the informational variables underlying the judgments provided was therefore restricted to the horizontal plane. A geometrical analysis identifies two optical angles as potentially informative: the optical size of the ball, captured by the angle $\varphi$, and the optical lateral bearing direction of the ball, captured by the angle $\theta$ (Bootsma \& Craig, 2002; Bootsma et al., 1997). Along with their respective rates of change, these angles are inextricably linked. For an object that is on collision course with an observer, the angle $\varphi$ (and its rate of change $\dot{\varphi}$ ) will increase rapidly, whereas the angle $\theta$ will remain unchanged ( $\dot{\theta}$ being 0 ). For an object moving laterally relative to the observer, the inverse is true. The information used to anticipate the arrival point could therefore be contained in the pattern of change of the angles $\varphi$ and $\theta$ during an event.

For a rectilinear trajectory, the dimensionless ratio of $\dot{\theta}$ over $\dot{\varphi}$ provides an estimate of future passing distance in ball-size units (Bootsma, 1991; Peper et al., 1994; Regan $\&$ Kaushal, 1994). On the basis of this logic, we identified two other dimensionless candidate variables: $(\dot{\theta} / \theta) /(\dot{\varphi} / \varphi)$, which corresponds to the ratio of the (inverse) tau functions of $\theta$ and $\varphi$, and $(\dot{\theta} / \dot{\varphi}) *(\theta / \varphi)$, which corresponds to the product of velocity and angle ratios. These three candidate variables were tested by regressing the percentage of "goal" judgments for each condition onto the values of the candidate information variables, using a logistic (S-shaped) function.

In a first analysis, we used the mean percentage of goal responses provided by the participants in Experiments 1 and 2, for a total of $(48+96=) 144$ different conditions.

The tau ratio $(\dot{\theta} / \theta) /(\dot{\varphi} / \varphi)$ clearly did not correspond to the information used, since it explained only $8.3 \%$ of the total variance in the percentage goal responses. The velocity ratio $\dot{\theta} / \dot{\varphi}$ did a better job, allowing $63.0 \%$ of the variance to be explained (see Table 1). However, the candidate explaining most of the variance $(82.5 \%)$ was the product of the velocity and angle ratios, $(\dot{\theta} / \dot{\varphi}) *(\theta / \varphi)$. Figure 2 presents the percentage of goal responses as a function of this informational variable.

For each of the three ball departure positions, Figure 3 presents the actual percentage of goal judgments made by participants for each arrival position (\% goal), along with the percentage of goal responses that are predicted by the informational variable $(\dot{\theta} / \dot{\varphi}) *(\theta / \varphi)$. Although the prediction is not perfect, this series of graphs shows how reliance on this information variable leads to the patterns of results obtained under the different spin conditions, with the prediction closely mirroring the qualitative aspects of the data.

In order to compare the groups of experts and novices, we reran the analyses, this time applying the logistic regression to the data of each individual participant separately. On the basis of the individual participant fits, we calculated the average values of the coefficient of determination $\left(R^{2}\right)$ and the average equation parameters resulting from the fits (corresponding to the critical value of the information variable in which $50 \%$ goal responses are predicted, and to the slope of the function at that point that describes the sharpness of the transition from goal to no-goal responses). The results are presented in Figure 4.

When comparing the $R^{2}$ and equation parameter values of the averaged individual participant data (dark bars in Figure 4A) with those obtained by fitting the group means (gray bars in Figure 4A), statistically significant

Table 1

$R^{2}$ Values of the Fits for the Different Information Variables Considered

\begin{tabular}{|c|c|c|c|c|c|}
\hline & \multirow[b]{2}{*}{$N$ Data Points } & \multirow{2}{*}{$\begin{array}{c}\text { Angle } \\
\text { Velocity Ratio } \\
\dot{\theta} / \dot{\varphi}\end{array}$} & \multirow{2}{*}{$\begin{array}{c}\text { Tau Ratio } \\
(\dot{\theta} / \theta) /(\dot{\varphi} / \varphi)\end{array}$} & \multicolumn{2}{|c|}{$\begin{array}{c}\text { Compound } \\
\text { Information Variable } \\
(\dot{\theta} / \dot{\varphi}) *(\theta / \varphi)\end{array}$} \\
\hline & & & & Individual & Mean \\
\hline GK-1 & 48 & & & .700 & .728 \\
\hline FP-1 & 48 & & & .657 & .701 \\
\hline NOV-1 & 48 & & & .726 & .860 \\
\hline MEAN-1 & 48 & .570 & .138 & & .783 \\
\hline NOV-2 & 96 & .663 & .065 & .776 & .854 \\
\hline All & 144 & .630 & .083 & & .825 \\
\hline
\end{tabular}

Note-For the angle velocity ratio and the tau ratio, the mean results per group (MEAN-1) are presented. For the compound information variable, the means of individual fits (Individual) and the value for the fit to the group average (Mean) for the different groups (Experiment 1: GK-1, FP-1, NOV-1) are also presented. The last row represents the results for the fit of the group averages (i.e., MEAN-1 for Experiment 1 and NOV-2 for Experiment 2) for all $48+96=144$ data points. 
A

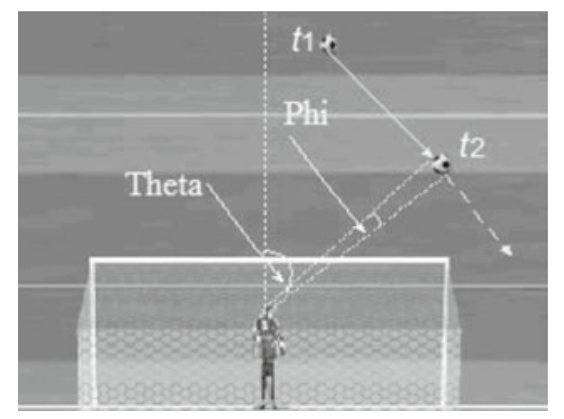

B

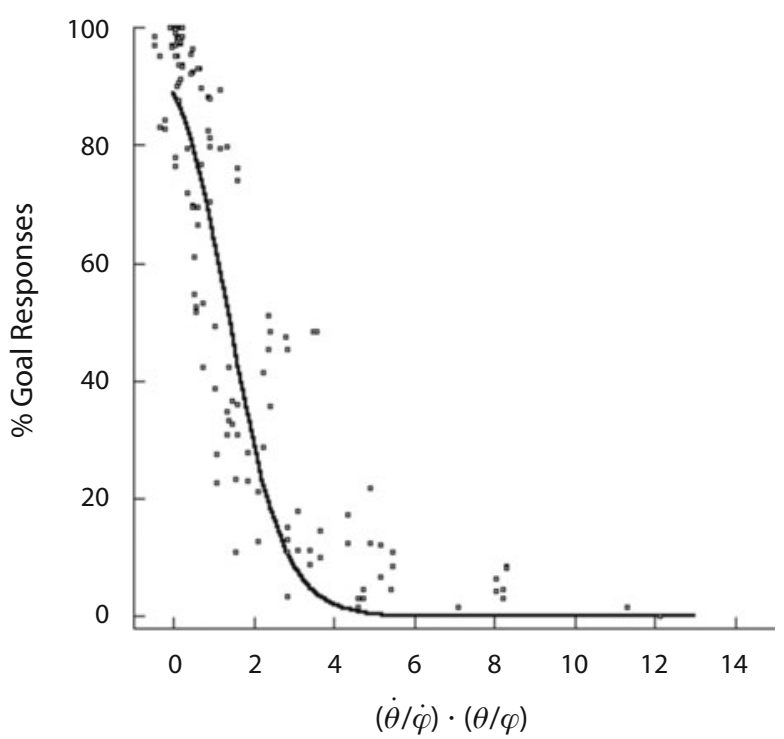

Figure 2. (A) Geometric representation of the angles phi $(\varphi)$ and theta $(\theta)$ used in the informational variable analysis with respect to the observer's position. $t_{1}$ and $t_{2}$ represent two different ball positions in time. (B) Relationship between the values of the compound variable $[(\dot{\theta} / \dot{\varphi}) *(\theta / \varphi)]$ calculated at the moment the ball vanished and the percentage of "goal" responses. Logistic regression of the 144 data points using percent goal $=100 /(1+$ $e^{-k(c-\text { info) })}$ fitted to the data explains $82.5 \%$ of the variance. In this equation, $c$ represents the critical value of the information value corresponding to $50 \%$ goal responses, and $k$ represents the slope of the function at this point (i.e., the sharpness of the transition).

differences were found only for the $R^{2}$ values of the novice groups $[t(12)=3.9, p<.05$, for the NOV -1 group, and $t(7)=3.6, p<.05$, for the NOV-2 group]. The slope and critical value parameters did not differ significantly between the individual participant fits and the fits of the group means (all $t \mathrm{~s}<2.0$; see Figures $4 \mathrm{~B}$ and $4 \mathrm{C}$ ). Thus, as was to be expected, fitting individual participant data rather than group data gave rise to a (moderate) lowering of the coefficient of determination of the fits, which was significant only for the two novice groups. However, fitting individual participant data rather than group data did not lead to significant changes in the resulting equation parameters.

Using the individual participant data, we compared groups through univariate ANOVAs. These ANOVAs did not reveal any statistically significant between-group differences for $R^{2}[F(3,57)=2.5$, n.s. $]$, slope $[F(3,57)=2.3$, n.s.], or critical values $[F(3,57)=1.4$, n.s. $]$.

\section{DISCUSSION}

Sidespin was found to affect the anticipatory judgments of top-level professional soccer players, regardless of whether they were field players or goalkeepers. Craig et al. (2006) suggested that this was due to an inherent limitation of the human visual system, which is incapable of dealing with the ever-varying accelerative influence of the Magnus force created by spin. In line with this suggestion, the present series of experiments revealed that the non-soccer-players' anticipatory judgments were also significantly influenced by the laterally deviating ball trajectory (caused by sidespin). In the presence of spin, the resulting ball trajectory systematically influenced the judgments made by both professional players and nonsoccer-players.

The fact that experts and novices alike are "fooled" by where these bending free kicks will eventually end up suggests that all groups of participants are attuned to the same informational variable(s) that provide correct responses in some conditions and erroneous responses in others. Focusing on the informational variable that provides the best prediction of response patterns, an analysis of both the information value corresponding to the critical point (separating goal and no-goal responses) and the sharpness of the goal/no-goal transition did not reveal any statistically significant differences between the groups of participants, regardless of whether they were experts (FP-1 and GK-1) or novices (NOV-1 and NOV-2). Together, these findings imply that expertise in soccer does not lie in the ability to make precise anticipatory judgments, but that it may-as previous research suggests - become apparent when perception is coupled to action (Farrow \& Abernethy, 2003; Oudejans, Michaels, Bakker, \& Dolné, 1996).

Under the conditions of Experiment 1, the ball always departed from the same position, located straight ahead of the center of the goalmouth at which the participants were situated. The use of two supplementary departure points in Experiment 2, positioned $4 \mathrm{~m}$ to the left and to the right of the central departure point used in Experiment 1, allowed for a larger range of conditions in identifying the optical information variable used when judgments are made about whether the ball would enter into the goalmouth or 


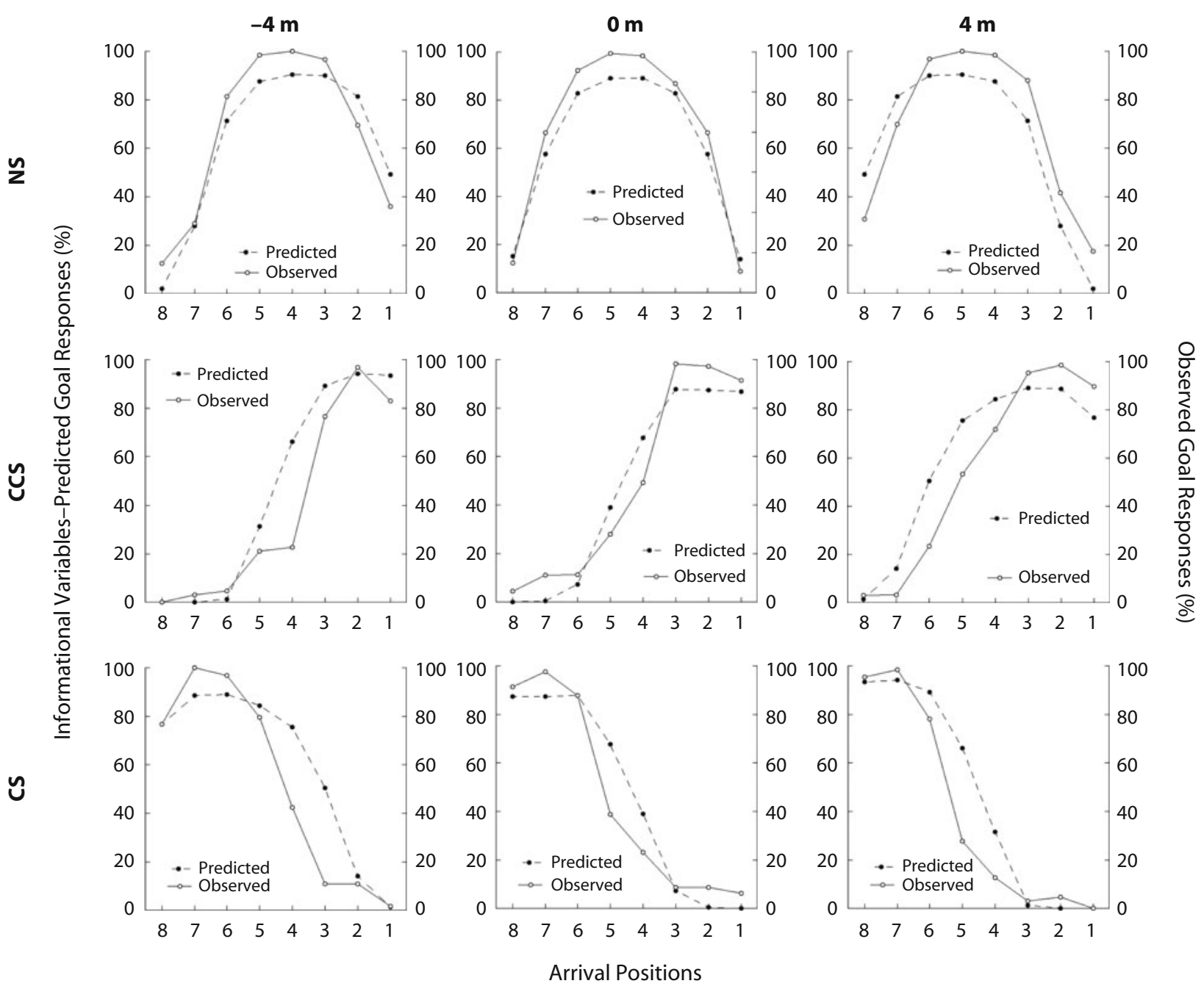

Figure 3. This series of graphs illustrates the similarities between the mean goal responses that would have ensued if the participants were using the compound information $(\dot{\theta} / \dot{\varphi}) *(\theta / \varphi)$ (predicted \% goal responses $=$ dashed line/solid circles) and the mean goal responses observed (observed \% goal responses $=$ solid line/open circles). The compound variable $(\dot{\theta} / \dot{\varphi}) *(\theta / \varphi)$ explains the largest percentage of variance in the observed data. The three columns represent the different departure positions $(-4,0$, and $+4 \mathrm{~m})$, whereas the rows show the corresponding spin conditions (NS, no spin; CCS, counterclockwise spin; CS, clockwise spin).

not. Bootsma (1991; see also Peper et al., 1994) suggested that the ratio of $\dot{\theta}$ over $\dot{\varphi}$ could provide useful spatial information with respect to the future arrival position, since this ratio specifies the lateral passing distance in ball-size units for a rectilinear trajectory. However, the analysis of the responses obtained for the 144 different conditions in the present series of experiments indicates that the velocity ratio $\dot{\theta} / \dot{\varphi}$ may not be isolated as such. Instead, the best-fitting model shows that this velocity ratio is complemented by an aspect ratio of $\theta / \varphi$.

The use of a multiplicative compound variable $(\dot{\theta} / \dot{\varphi}) *(\theta / \varphi)$ is in accordance with recent studies that have shown that the neuronal firing response to upcoming collisions is best described by the product of the object's optical size $(\varphi)$ and its rate of change $(\dot{\varphi})$ (Gabbiani, Krapp, Koch, \& Laurent, 2002). Other studies have likewise shown that animals (Sun \& Frost, 1998) and humans (Bootsma \& Craig, 2002) are sensitive to particular pat- terns ( $\tau \mathrm{s}$; Lee, 1998) of change of the angle $\varphi$. The focus of these studies has been on the salience of $\varphi$ (and other optical angles) in collision detection, whereas the present study goes one step further in looking at the combination of expansion and lateral displacement when spatial judgments are made. The results clearly demonstrated that arrival position judgments were not made on the basis of the ratio of the $\tau$ functions of angles $\varphi$ and $\theta$.

Successful interception is dependent on some kind of knowledge about where something is going, which may help explain why top-level goalkeepers are sometimes duped by curving free kicks. The results of the present study show clearly that the lateral deflection in the ball's flight path caused by sidespin has profound consequences for a goalkeeper's anticipation of the ball's future point of arrival. The analysis of potential informational variables onto which participants may latch suggests that an optic variable combining (changes in) optical size and (changes 


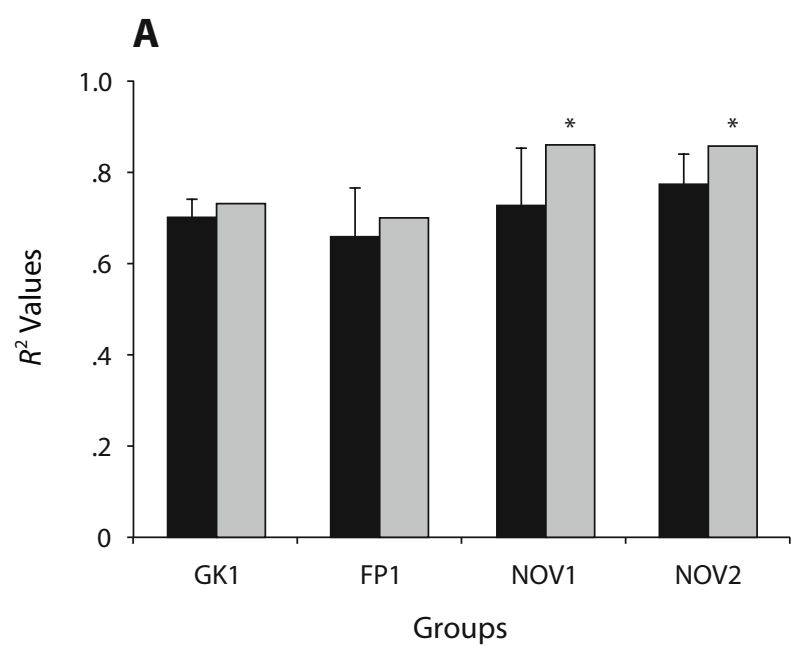

B
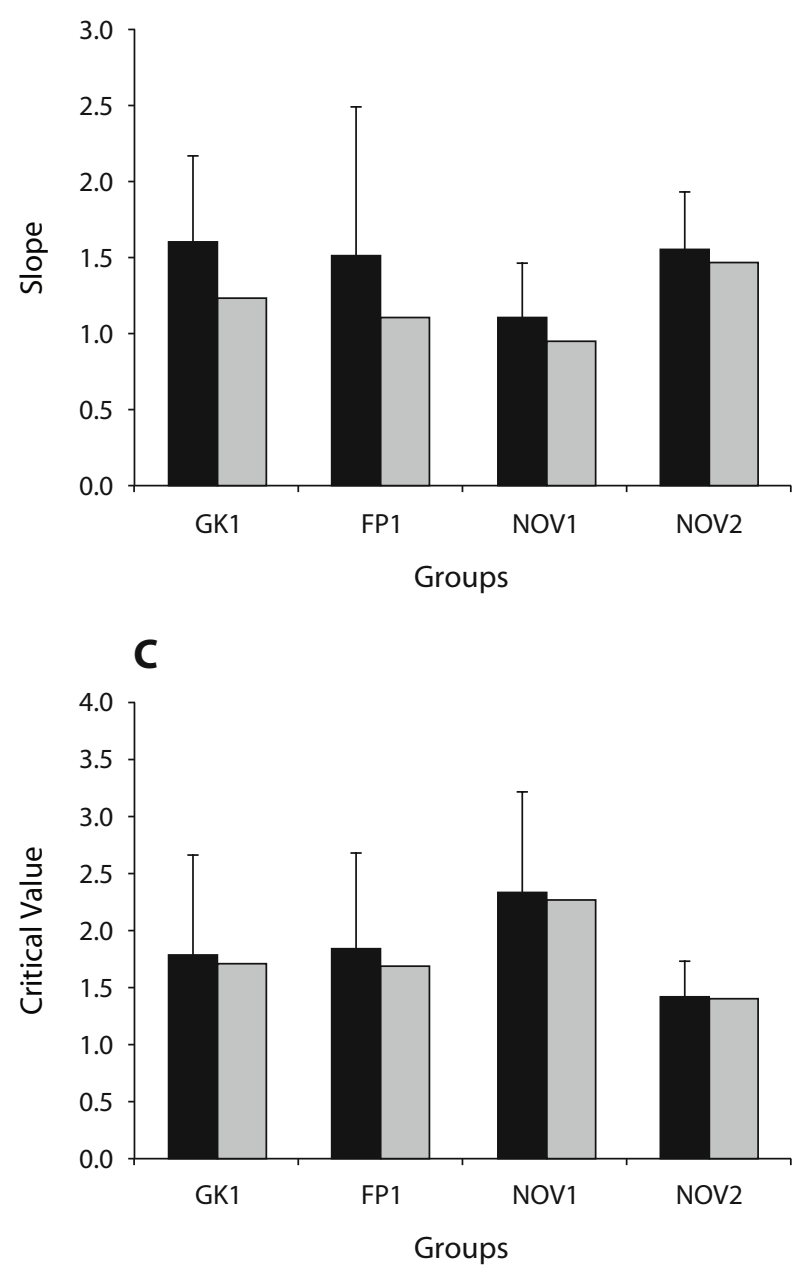

Figure 4. Results of the logistic fit for both individual participant data and group data for the compound information variable $(\dot{\theta} / \dot{\varphi}) *(\theta / \varphi)$. Black bars represent the parameter means of individual fits (error bars represent between-participants standard deviation) and gray bars represent parameter values for the fit of averaged group scores. (A) Results for the $R^{2}$ values. (B) Slopes. (C) Critical value parameter. " Significant differences. in) optical bearing allowed participants to make fairly accurate predictions about where a nonspinning ball was going, whereas it led to considerable errors in their predictions of the future arrival point when the ball flight was subjected to a lateral deflection.

Since even the world's best players are prone to error, one therefore has to assume that expertise resides more in the way in which task-relevant actions are organized (Michaels \& Oudejans, 1992; Shaffer, McBeath, Roy, \& Krauchunas, 2003) than in anticipatory perceptual spatial judgment capabilities. Although our present results beg the question concerning the information used to guide a goalkeeper's actions (Farrow \& Abernethy, 2003; Oudejans et al., 1996), the identification of these operative information-movement relationship(s) (McLeod \& Dienes, 1996; Montagne, Laurent, Durey, \& Bootsma, 1999) is certainly an area that needs to be explored in future studies.

\section{AUTHOR NOTE}

Address correspondence to C. M. Craig, School of Psychology, Queen's University Belfast, David Keir Building, 18-30 Malone Rd., Belfast BT7 1NN, Northern Ireland (e-mail: cathy.craig@qub.ac.uk).

\section{Note-Accepted by the previous editorial team, when Thomas H. Carr was Editor.}

\section{REFERENCES}

Abernethy, B., Gill, D. P., Parks, S. L., \& Packer, S. T. (2001). Expertise and the perception of kinematic and situational probability information. Perception, 30, 233-252.

Bootsma, R. J. (1991). Predictive information and the control of action: What you see is what you get. International Journal of Sport Psychology, 22, 271-278.

Bootsma, R. J., \& Craig, C. M. (2002). Global and local contributions to the optical specification of time to contact: Observer sensitivity to composite tau. Perception, 31, 901-924.

Bootsma, R. J., Fayt, V., ZaAL, F. T. J. M., \& Laurent, M. (1997). On the information-based regulation of movement: What Wann (1996) may want to consider. Journal of Experimental Psychology: Human Perception \& Performance, 23, 1282-1289.

Bray, K., \& Kerwin, D. G. (2003). Modelling the flight of a soccer ball in a direct free kick. Journal of Sports Sciences, 21, 75-85.

Carré, M. J., Asai, T., Akatsuka, T., \& Haake, S. J. (2002).The curve kick of a football: II. Flight through the air. Sports Engineering, 5, 193-200.

Craig, C. M., Berton, E., Rao, G., Fernandez, L., \& Bootsma, R. J. (2006). Judging where a ball will go: The case of curved free kicks in football. Naturwissenschaften, 93, 97-101.

DE Mestre, N. (1990). The mathematics of projectiles in sport. Cambridge: Cambridge University Press.

FARrow, D., \& ABERnethy, B. (2003). Do expertise and the degree of perception-action coupling affect natural anticipatory performance? Perception, 32, 1127-1139.

Gabbiani, F., Krapp, H. G., Koch, C., \& Laurent, G. (2002). Multiplicative computation in a visual neuron sensitive to looming. Nature, 420, 320-324.

Goulet, C., Bard, C., \& Fleury, M. (1989). Expertise differences in preparing to return a tennis serve: A visual information processing approach. Journal of Sport \& Exercise Psychology, 11, 382-398.

GraY, R., \& REgaN, D. M. (2006). Unconfounding the direction of motion in depth, time to passage and rotation rate of an approaching object. Vision Research, 46, 2388-2402.

HARRIS, J. M., \& DRGA, V. F. (2005). Using visual direction in threedimensional motion perception. Nature Neuroscience, 8, 229-233.

LeE, D. N. (1998). Guiding movement by coupling taus. Ecological Psychology, 10, 221-250. 
LeE, D. N., \& Young, D. S. (1985). Visual timing of interceptive action. In D. J. Ingle, M. Jeannerod, \& D. N. Lee (Eds.), Brain mechanisms and spatial vision (pp. 1-30). Dordrecht: Martinus Nijhoff.

McLeod, P., \& Dienes, Z. (1996). Do fielders know where to go to catch the ball or only how to get there? Journal of Experimental Psychology: Human Perception \& Performance, 22, 531-543.

Menta, R. D. (1985). Aerodynamics of sports balls. Annual Review of Fluid Mechanics, 17, 151-189.

Michaels, C. F., \& Oudejans, R. R. D. (1992). The optics and actions of catching fly balls: Zeroing out optical acceleration. Ecological Psychology, 4, 199-222.

Montagne, G., Laurent, M., Durey, A., \& Bootsma, R. [J.] (1999). Movement reversals in ball catching. Experimental Brain Research, 129, 87-92.

Morya, E., Ranvaud, R., \& Pinheiro, W. M. (2003). Dynamics of visual feedback in a laboratory simulation of a penalty kick. Journal of Sport Sciences, 21, 87-95.

Oudejans, R. R. D., Michaels, C. F., Bakker, F. C., \& Dolné, M. A. (1996). The relevance of action in perceiving affordances: Perception of catchableness of fly balls. Journal of Experimental Psychology: Human Perception \& Performance, 22, 879-891.
Peper, L., Bootsma, R. J., Mestre, D. R., \& Bakker F. C. (1994). Catching balls: How to get the hand to the right place at the right time. Journal of Experimental Psychology: Human Perception \& Performance, 20, 591-612.

Regan, D. [M.], \& Kaushal, S. (1994). Monocular discrimination of the direction of motion in depth. Vision Research, 34, 163-177.

Shaffer, D. M., McBeath, M. K., Roy, W. L., \& Krauchunas, S. M. (2003). A linear optical trajectory informs the fielder where to run to the side to catch fly balls. Journal of Experimental Psychology: Human Perception \& Performance, 29, 1244-1250.

Sun, H., \& Frost, B. J. (1998). Computation of different optical variables of looming objects in pigeon nucleus rotundus neurons. Nature Neuroscience, 1, 296-303.

Williams, A. M., \& ERIcsson, K. A. (2005). Perceptual-cognitive expertise in sport: Some considerations when applying the expert performance approach. Human Movement Science, 24, 283-307.

(Manuscript received October 25, 2006; revision accepted for publication November 24, 2008.) 